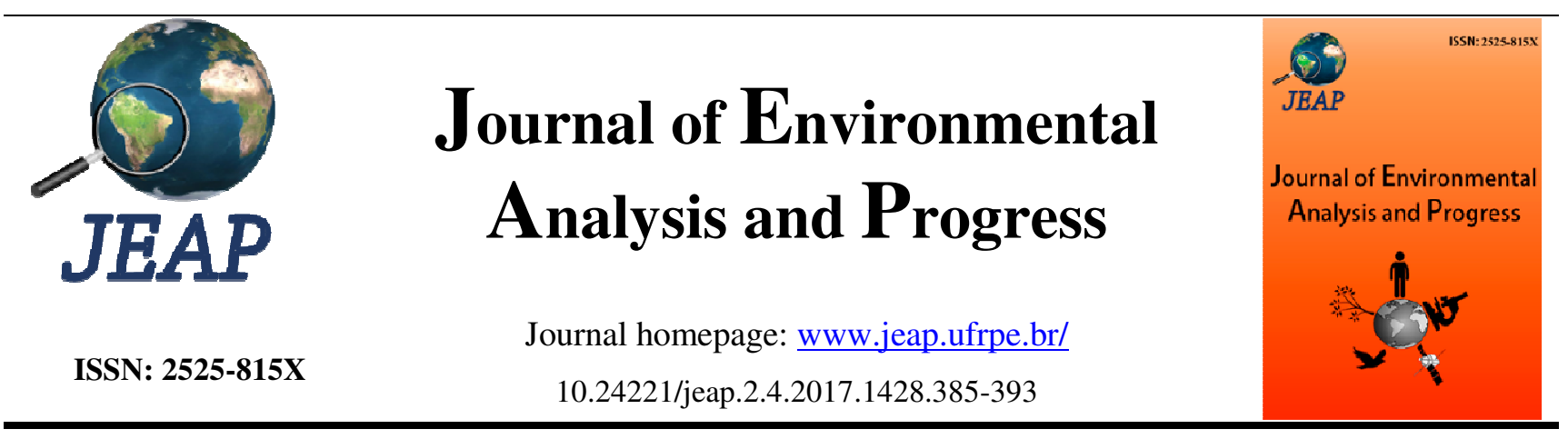

\title{
Downscaling dinâmico de precipitação e veranicos no estado do Ceará
}

\section{Dynamic downscaling of precipitation and dry spells in Ceará State}

\author{
José Maria Brabo Alves ${ }^{\mathrm{a}}$, Emerson Mariano da Silva ${ }^{\mathrm{a}}$, Cláudia Patrícia Rickes ${ }^{\mathrm{b}}$ \\ a Universidade Estadual do Ceará-UECE, Departamento de Física, Fortaleza, Ceará, Brasil. E-mail: \\ braboalves@gmail.com, emerson@uece.br. \\ b Instituto Nacional de Meteorologia-INMET, Brasília, DF, Brasil, claurickes@ hotmail.com.
}

\begin{tabular}{ll}
\hline A R T I C L E I N F O & A B S T R A C T \\
\cline { 1 - 3 } Recebido 26 Jun 2017 & The semi-arid region of Northeast Brazil (NEB) is known to be an extremely \\
Aceito 14 Ago 2017 & oscillating region from the climatic point of view, due to the temporal and spatial \\
Publicado 15 Ago 2017 & variability of precipitation. The objective of this work was analyzed the internal \\
seasonal and interannual variability of the precipitations, through dry spells, in the & State of Ceará. This behavior is directly influenced by the Sea Surface Temperature \\
& (SST) of the Pacific and Atlantic oceans. The RSM97 Regional Spectral Model, \\
& through the dynamic downscaling technique, was used to quantify variations using \\
& dry spells events (consecutive days with rainfall below 2 mm, occurred during the \\
& rainy season of the state), from 1974 to 2012, through FUNCEME daily \\
& precipitation data, atmospheric numerical model simulations and SST anomalies \\
& (ENSO phases). The results were presented in two stages, first diagnostic mode \\
& (with SST anomalies observed from 1974 to 2000) and the second prognostic mode \\
& (with persistent SST anomalies from 2002 to 2012). It is concluded that the RSM97 \\
model, predicting drought periods during the rainy season, is reasonable, to a great \\
part the results the model underestimated the number of dry spells in relation to \\
those observed, but represented the signal of the observations of the events which \\
indicates that its physical characteristics influenced the quantification of \\
precipitation and its spatial distribution in the state \\
Keywords: Dry spells, modeling, correlation.
\end{tabular}

Keywords: Dry spells, modeling, correlation. 
em quantificar a precipitação e sua distribuição espacial no Estado.

Palavras-Chave: Veranicos, modelagem, correlação.

\section{Introdução}

A região semiárida do Nordeste do Brasil (NEB) é conhecida por ser extremamente oscilante do ponto de vista climático, devido à variabilidade temporal e espacial da precipitação, sendo a água um condicionante socioeconômico. Os fatores que influenciam o comportamento da precipitação no NEB são inúmeros, um deles é a variabilidade térmica da superfície dos oceanos, principalmente o Pacífico e o Atlântico, e suas interações com a atmosfera. "Em um estudo pioneiro, Walker (1928) documentou uma notável coincidência do aquecimento anômalo das águas superficiais no Pacífico equatorial e as secas do Nordeste do Brasil" (Andreoli \& Kayano, 2007).

A partir desta constatação pesquisadores, têm destacado particularidades da variabilidade sobre a região do nordeste do país e suas correlações com os padrões térmicos das regiões oceânicas tropicais. Entre eles, destacamos os estudos de Moura \& SHukla (1981), Hastenrath (1984), Nobre \& Shukla (1996), Alves et al. (1997, 2005), Uvo (1989), Souza; Alves \& Repelli (1998), Servain (1991), Lucena, Servain \& Gomes Filho (2011) e Alves (2012).

O modo de variabilidade dominante no Pacífico é o fenômeno ENOS (El Niño-Oscilação Sul), que consiste em uma componente oceânica, o El Niño, e outra atmosférica, a Oscilação Sul (OS). Na sua componente oceânica, caracteriza-se pelo aquecimento das anomalias da temperatura da superfície do mar, no Oceano Pacífico Equatorial Central e Leste - EI Niño (fase quente). A fase do ENOS resfriado ou episódio de La Niña apresenta os padrões de anomalias opostos (Kousky \& Ropelewski, 1989).

A componente atmosférica do ENOS está acoplada dinamicamente ao Índice de Oscilação Sul (IOS), que é definido pela diferença entre a pressão atmosférica ao nível do mar entre o Pacífico Central (Taiti) e o Pacífico do Oeste (Darwin). O IOS apresenta valores negativos em anos de EI Niño (fase quente) e positivos em anos de La Niña (fase fria) (Alves \& Repelli, 1992). Portanto, valores positivos significam resfriamento do Pacífico e os negativos, aquecimento (Lucena, Servain \& Gomes Filho, 2010). Em geral, em anos de El Niño (La Niña) as chuvas tendem a ser escassas (regulares) na região semiárida do NEB.

Considerando o Oceano Atlântico, enfatizamos o dipolo descrito por Moura \& Shukla (1981) e Servain (1991). O Padrão de Dipolo, ou gradiente meridional de anomalia da Temperatura da Superfície do Mar (TSM), é um modo de variabilidade oceano-atmosfera de grande escala mais importante quanto a variabilidade climática interanual sobre a Bacia do Atlântico Tropical, que ocorre durante o outono austral (março-abril-maio), período coincidente com a estação chuvosa no semiárido nordestino (Nobre \& Shukla, 1996).

Segundo Alves; Ferreira \& Campos (2005), a Zona de Convergência Intertropical (ZCIT) constitui-se no principal sistema meteorológico gerador de chuvas na região do semiárido nordestino, durante os meses da estação chuvosa (fevereiro a maio). $\mathrm{Na}$ fase positiva (negativa) do padrão do dipolo, observam-se anomalias positivas (negativas) de TSM nas bacias norte (sul) do Atlântico Tropical. Nessa situação, a ZCIT posiciona-se sobre a região do Atlântico Norte, mantendo-se afastada do NEB. Em anos da fase negativa do dipolo de TSM, estas características são contrárias, consequentemente, as chuvas são frequentes na região (Moura \& Shukla, 1981; Nobre \& Shukla, 1996).

Andreoli \& Kayano (2007) constatam que a influência do Atlântico Tropical na variabilidade de precipitação do NEB é mais marcante para a variabilidade de TSM do Atlântico Tropical Sul (ATS), quando comparada com a do Atlântico Tropical Norte (ATN), independente do ENOS. Esse resultado é consistente com os estudos de Moura \& Shukla (1981) e Lucena, Servain \& Gomes Filho (2011).

Com relação às probabilidades de ocorrência de veranicos no Ceará, Repelli \& Alves (1996) diagnosticaram que se pode esperar, durante a estação chuvosa, que períodos longos (acima de 20 dias) sejam mínimos, inclusive nos municípios localizados nas áreas mais áridas do Estado (Sertão Central/Inhamuns). Esta característica mostra que, em média, quando as chuvas se tornam regulares, provocadas principalmente pela influência da ZCIT, os períodos de estiagem prolongada sobre o Estado tendem a ser mais escassos.

$\mathrm{O}$ veranico indica uma irregularidade temporal da precipitação, que é uma característica bastante comum no regime de chuva cearense, principalmente, em anos cuja estação chuvosa apresenta precipitação abaixo da média, ou seja, quando a ZCIT se posiciona acima da linha do equador e/ou sobre o ATN durante a estação chuvosa (Uvo, 1989). Sun et al. (2005) mostraram que há potencial em seus resultados para investigar a variabilidade pluviométrica intrassazonal no Estado do Ceará, através da modelagem numérica regional, através de 
downscaling dinâmico. Alguns autores, como Nobre et al. (2001), Alves et al. (2005) e Pereira et al. (2015) mostram que a técnica tem um melhor desempenho em escalas menore, das simulações advindas de modelagem dinâmica de grande escala, os chamados modelos atmosféricos globais.

No atual contexto de mudanças climáticas globais, o quinto relatório do Painel Intergovernamental de Mudanças Climáticas (IPCC) inferiu que região semiáridas como parte do Nordeste do Brasil, poderá sofrer com períodos de estiagens mais freqüentes e com o aumento da temperatura a superfície. O objetivo do estudo foi aprimorar o conhecimento sobre a variabilidade interanual e intrassazonal da precipitação no Estado do Ceará, durante a estação chuvosa (fevereiro a maio), com ênfase nos veranicos, além de investigar o potencial de simulações e previsões desses veranicos usando a técnica de redução de escala (downscaling dinâmico).

\section{Material e Métodos}

O Estado do Ceará está inserido no Nordeste brasileiro, situado numa posição tropical entre $2^{\circ} 46^{\prime} 30^{\prime \prime}$ e $7^{\circ} 52^{\prime} 15^{\prime \prime}$ de latitude sul e $37^{\circ}$ $14^{\prime} 54^{\prime \prime}$ e $41^{\circ} 24^{\prime} 45^{\prime \prime}$ de longitude ocidental, possui 184 municípios, dos quais aproximadamente 150 fazem parte da região semiárida do Brasil, na qual tem como característica marcante a irregularidade espacial e temporal das precipitações pluviométricas.

\section{Dados de precipitação}

Utilizou-se uma série histórica de dados diários de precipitação de mais de 100 postos pluviométricos do Ceará, referente ao período de fevereiro a maio, de 1974 a 2012, obtido através do banco dados da FUNCEME.

\section{Modelo climático regional}

Para precipitação diária simulada os dados usados foram de simulações climáticas (19742000) executadas na Fundação Cearense de Meteorologia e Recursos Hídricos (FUNCEME) via downscaling dinâmico. Utilizou o modelo Regional Spectral Model versão 97 (RSM97) de 1971-2000, forçado pelo modelo climático global, ECHAM4.5 (European Community Hamburg versão 4.5), nessa simulação a TSM usada foi a observada, essa técnica é chamada previsão climática "perfeita" (diagnóstico). E para as simulações em modo chamado de previsão (prognóstico) utilizou-se a condição de TSM persistida no período de 2002-2012 das simulações geradas no mês de janeiro, para os meses de fevereiro a maio (FMAM).
Maiores detalhes sobre a característica da rodada via downscaling do modelo RSM/97 acoplado ao modelo ECHAM4.5 podem ser encontrados em Alves et al. (2003). Após a interpolação, usando o software GRADS (Grid Analysis and Display System) foram confeccionados mapas de isolinhas mostrando as comparações espacial e temporal da variação e características associadas a cada evento de veranico, entre os dados observados e modelados.

\section{Metodologia e estatística de avaliação dos resultados}

Considerou um veranico como sendo o número de dias consecutivos com chuva abaixo de $2 \mathrm{~mm} /$ dia. Após essa definição, foi classificado o número de eventos em função da quantidade de dias, escolhendo veranicos de 5, 10 e 15 dias. Também foram investigadas outras características como: o número de eventos de veranicos em anos de contrastes climáticos (anos de El Niño e La Niña e Neutros); a correlação entre os veranicos observados e simulados (RSM97) através do coeficiente de correlação de Pearson (r) e coeficiente de determinação $\left(\mathrm{R}^{2}\right)$; verificou-se, também, os erros médios entre os veranicos observados e simulados. A correlação foi calculada pela Equação 1 abaixo.

$r_{x y}=r=\frac{\sigma o v(X, Y)}{\sigma_{X}, \sigma_{Y}}$

onde $\mathrm{r}_{\mathrm{xy}}$ representa a correlação linear de Pearson entre a variável X e Y, cov $(X, Y)$ é a covariância entre duas variáveis $\mathrm{X}$ e $\mathrm{Y}, \sigma_{\mathrm{X}}$ e $\sigma_{\mathrm{Y}}$ são os desvios-padrão (variâncias) de $\quad X \quad$ e $\quad Y$, respectivamente.

Aplicou-se um teste estatístico para conhecer o grau real de ligação entre as variáveis analisadas, optou-se pela utilização do teste $t$ de Student Bilateral. Os valores de $r$ estão limitados entre $-1 \mathrm{e}+1$, onde o valor indica a direção da relação linear entre os dados. Valores extremos de $r$, geralmente na vizinhança tanto de -1 quanto de +1 , indicam uma relação relativamente forte, e valores próximos de 0 (zero) designam uma relação fraca ou quase inexistente, ou seja, significa que as duas variáveis são independentes linearmente uma da outra (Witte \&Witte, 2005).

Os anos foram classificados em El Niño, La Niña e Neutro (fase de neutralidade), baseados num limiar de $+/-0.5^{\circ} \mathrm{C}$, usando os dados de anomalias de TSM Comprehensive AtmosphericOcean Data Set (COADS), considerando apenas o período de fevereiro a maio. 


\section{Resultados}

Correlação entre veranicos observados $e$ simulados pelo RSM97

Calculou-se o coeficiente de correlação linear (r) entre os veranicos observados e os simulados do RSM97 de 5, 10 e 15 dias, para o Estado do Ceará (Figura 1). Destacou-se a relação entre os veranicos observados e os simulados no qual o coeficiente de correlação obteve magnitude forte, quando apresentou valores positivos (entre
$0,6$ e 0,9$)$ em parte da Serra da Ibiapaba (SI), Setor Norte (SN), Centro-Leste (CL), Região Metropolitana de Fortaleza (RMF), Cariri (RC) e Setor Central (SC) do Estado, assumindo que as variáveis estão diretamente relacionadas nessas regiões. As áreas correlacionadas são estatisticamente significativas a $95 \%$ segundo teste t-Student, e as áreas em branco no mapa não apresentaram significância estatística através do teste.
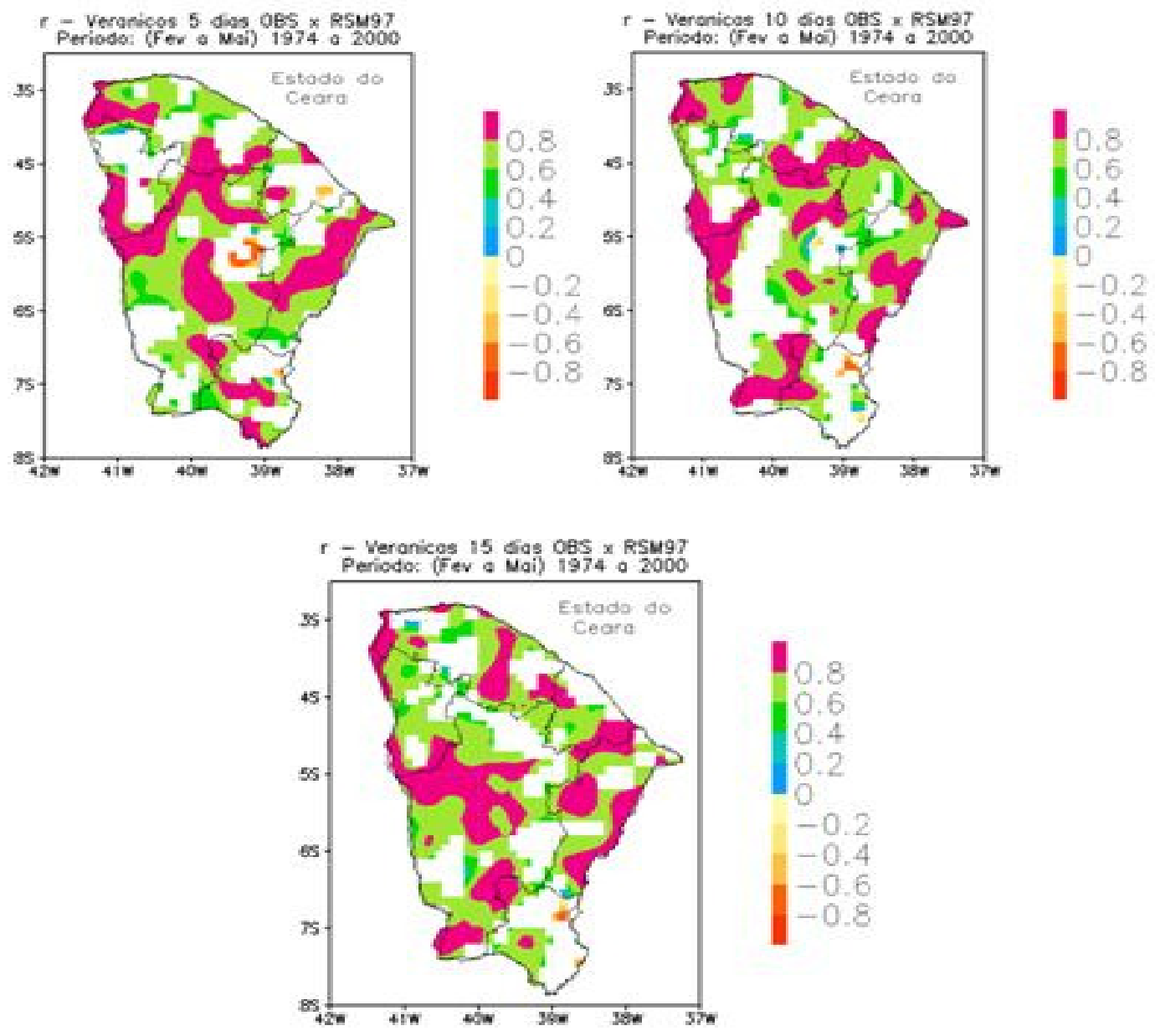

Figura 1. Correlação (r) entre os dados observados e os dados simulados (Regional Spectral Model - RSM97) de veranicos de 5, 10 e 15 dias, para o período dos meses de fevereiro a maio (FMAM), de 1974 a 2000. Foram plotadas apenas as áreas com correlação significativa 95\% segundo teste t Student.

Erro médio entre dados observados e simulados pelo RSM97

Para analisar o quanto a modelagem dinâmica superestima ou subestima quantitativamente os dados simulados em relação ao observado, utilizou-se a metodologia do erro médio, entre os meses FMAM de 1974-2000, para o modelo RSM97.
Os resultados do erro médio entre o número de eventos de veranicos observados e simulados RSM97 indicou, em grande parte do mapa, que o modelo subestimou as observações ocorridas durante o período, principalmente nos episódios de veranicos de 5 dias (Figura 2), consequentemente, isso indica que o modelo previu menor número de veranico para o período, concordando com as análises anteriores. 


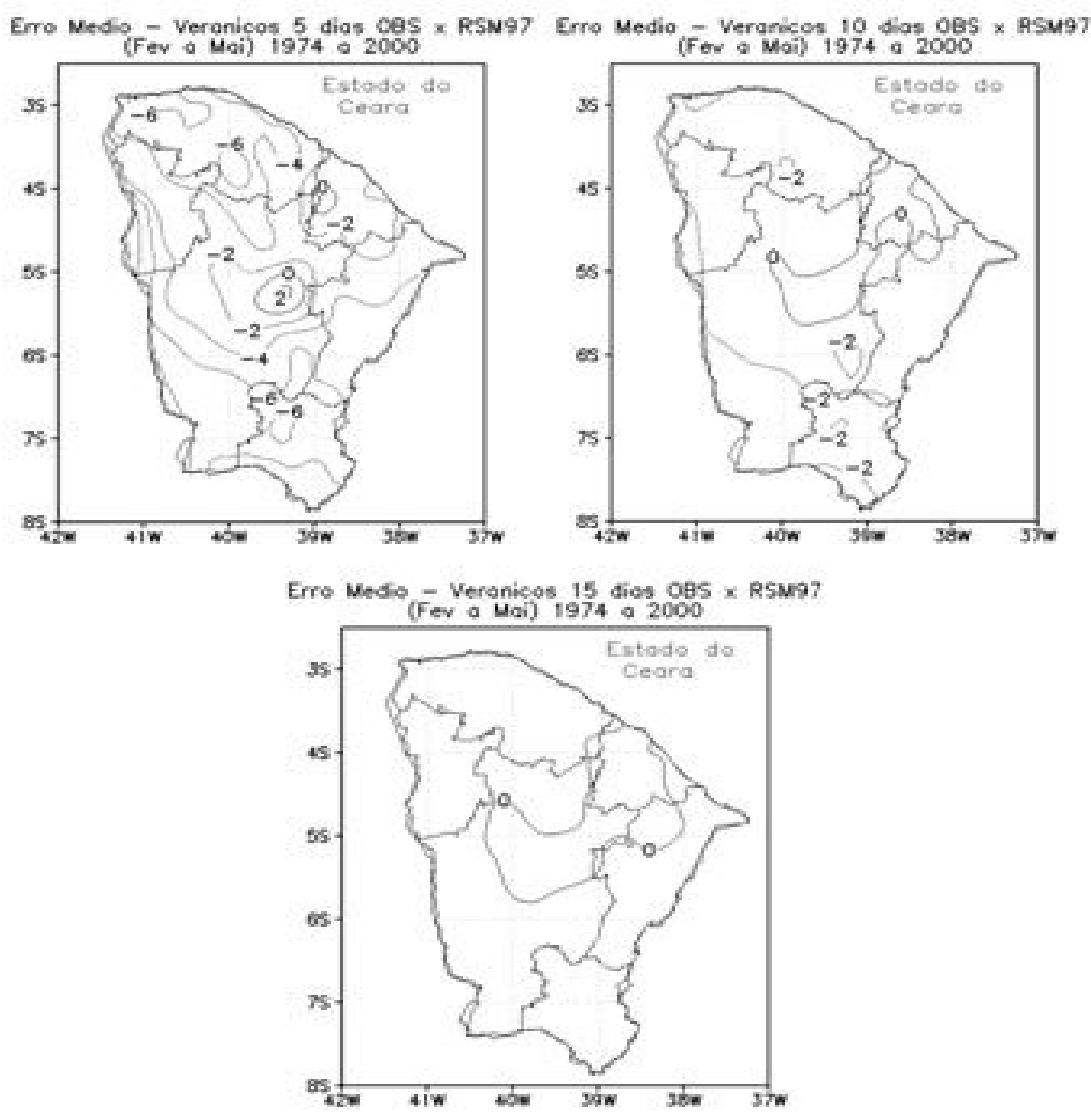

Figura 2. Erro Médio (número de eventos) entre observados e Regional Spectral Model-RSM97, de fevereiro a maio de 1974 a 2000 para veranicos de 5,10 e 15 dias.

Para as análises realizadas em modo de prognóstico, conclui-se que: nos resultados entre observados e RSM97 (com downscaling do modelo global e TSM persistida) destacam-se que, em anos de El Niño e La Niña, em geral, os resultados do modelo regional subestimam as observações (os números de veranicos calculados a partir dos dados observados). Apenas em anos Neutros, o modelo regional apresentou coerência com as observações. Nas correlações entre observados e RSM97 os resultados não registraram correlações significativas, para o erro médio o modelo seguiu subestimando as observações, exceto nos veranicos de 15 dias, e nos diagramas de dispersão, os resultados do $\mathrm{R}^{2}$ foram melhores principalmente nos Sertão Central e Setor Norte do Estado, porém com valores abaixo de 0,2 , para eventos de veranicos de 5 dias.

Resultados das análises modo prognóstico (20022012)

As análises em modo prognóstico possuem dados de 11 anos, período de 2002 a 2012, contendo a simulação em modo chamado de previsão, na qual agrega a média dos membros do modelo RSM97, que foram forçadas com anomalias de TSM, do mês de janeiro, de maneira persistida (PER), para os meses da quadra chuvosa no Estado do Ceará (FMAM).

Correlação entre veranicos observados $e$ simulados pelo RSM97

Nas análises de associação linear existente entre as variáveis, para eventos de veranicos de 5 , 10 e 15 dias, observados e previstos pelo modelo regional RSM97, para o Ceará, não foram observadas correlações significativas. Destaca-se que nos três eventos o coeficiente mostrou-se com pouca correlação sobre o Estado, apenas em pequenas áreas isoladas, oscilando entre positivo forte (valores superiores a 0,6). Entretanto, nas regiões do Estado, SN, SC, CL e RMF, às correlações foram negativas em pequenas áreas, Figura 3. As áreas correlacionadas são estatisticamente significativas a $95 \%$ segundo teste t-Student, e as áreas em branco nos mapas não apresentaram significância estatística através do teste. 


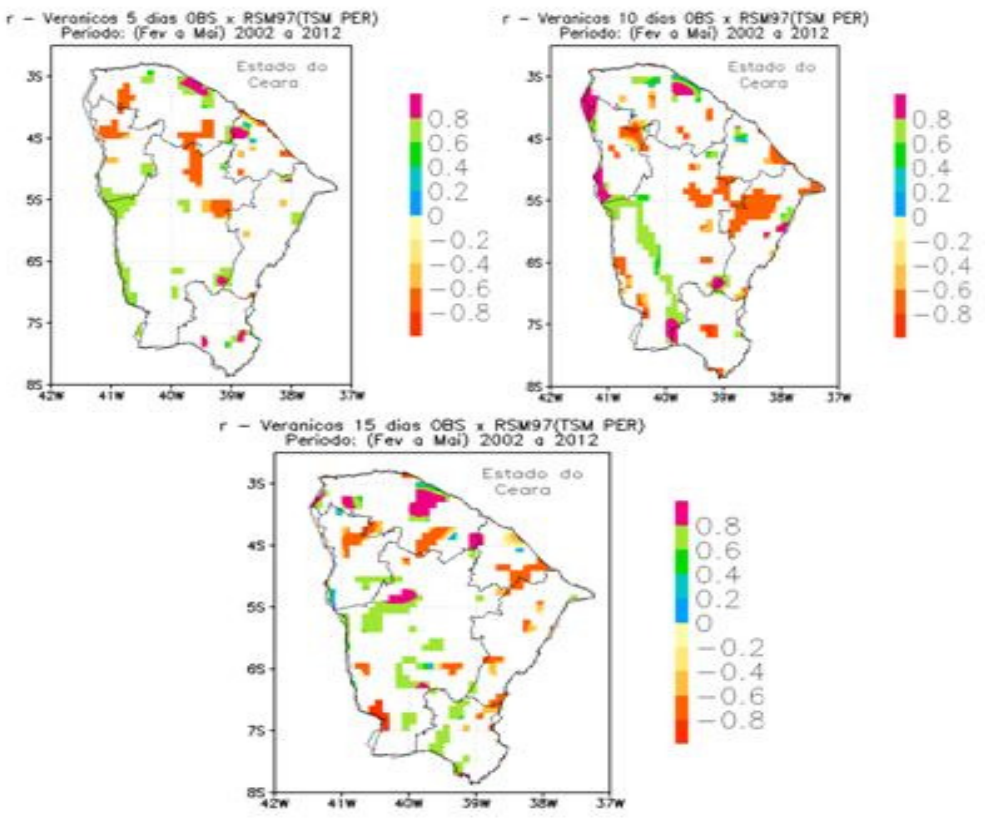

Figura 3. Correlação (r) entre os veranicos observados e previstos pelo Regional Spectral Model-RSM97 com Temperatura da Superfície do Mar (TSM) persistida (PER) para 5, 10 e 15 dias, para o período de FMAM de 2002 a 2012. Foram plotadas apenas as áreas com correlação significativa 95\% segundo teste $\mathrm{t}$ Student.

Erro médio entre dados observados e previstos pelo RSM7

O erro médio representa um indicativo de desempenho do modelo, ou seja, o quanto mais próximo ou distante o modelo previu em relação aos veranicos observados. Os resultados do erro médio entre o número de eventos de veranicos observados e simulados RSM97 (Figura 4) indicaram, em grande parte do mapa de veranicos de 5 dias, que o modelo subestimou as observações ocorridas durante o período, previu menor número de veranicos durante o período de 2002 a 2012. Para veranicos de 10 dias o erro mostrou-se pequeno, porém o modelo continuou subestimando o ocorrido, e por fim nos episódios de veranicos de 15 dias o erro foi nulo.

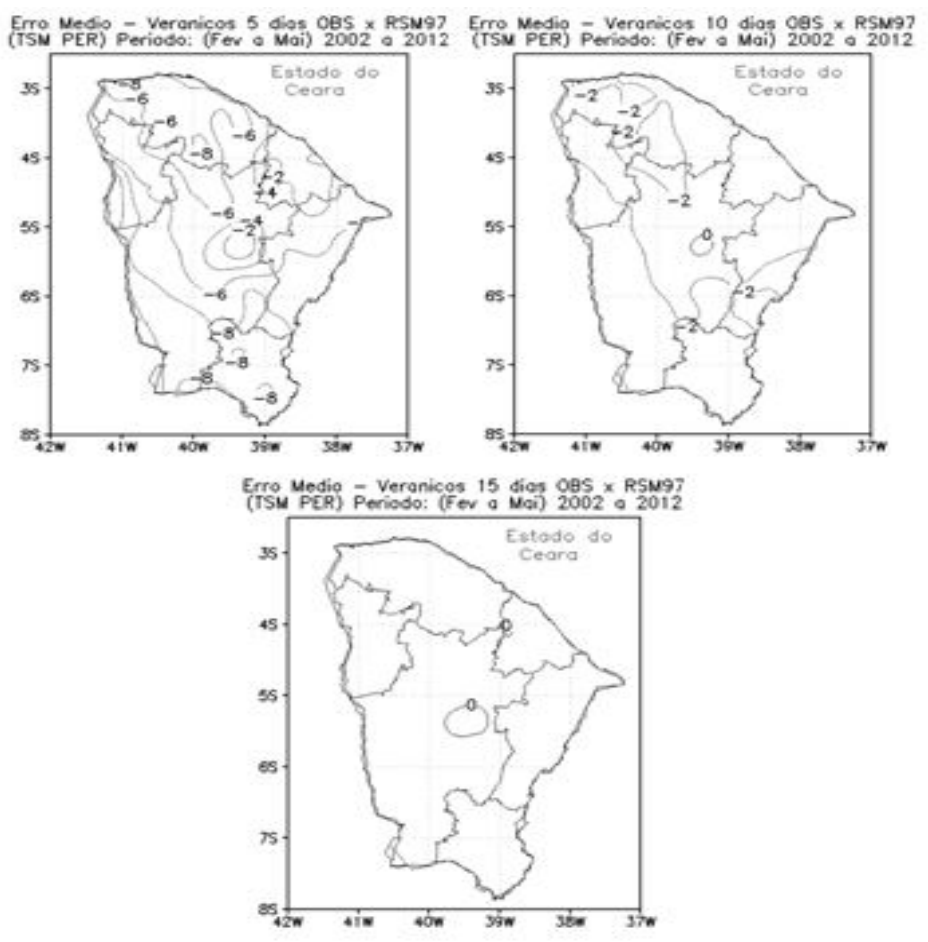

Figura 4. Erro médio (número de eventos) entre observados e Regional Spectral Model-RSM97, de fevereiro a maio de 2002 a 2012 para veranicos de 5, 10 e 15 dias. 


\section{Discussão}

De modo geral, o coeficiente de correlação apresentou melhor relação entre variáveis observadas e as simuladas pelo modelo RSM97, com coeficientes de moderado a forte (0.6 a 0.9), principalmente sobre o Setor Central do Estado. Esses resultados concordam com aqueles encontrados por Alves, Ferreira \& Campos (2005) e Pereira et al. (2015), que constataram correlações de 0,75 para o índice de seca na região central do Ceará.

Ribeiro Filho (2009) dentre outros autores descreveram que a precipitação na América do Sul tem um nível significativo de previsibilidade na maioria dos casos, usando como preditor a TSM do Pacífico e Atlântico Tropical. Logo, é altamente indicativo que a persistência de anomalias da TSM nesses oceanos tropicais mostra uma região fonte de previsibilidade de chuva em escala sazonal (Sun et al., 2006). Utilizando essas considerações, os resultados de simulações impulsionadas com a TSM prevista não foram calculados, pois o comportamento da TSM persistida mostra habilidade mais elevada e, portanto, mais confiável para esse tipo de estudo.

\section{Conclusão}

O modelo regional RSM97, mesmo alimentado por um modelo global forçado com as TSMs observadas, mostrou que subestima o número de veranicos ocorridos, durante a configuração de El Niño no Oceano Pacífico.

A utilização de métodos estatísticos, nas análises do erro médio, o modelo RSM97 subestimou as observações, destacando-se como satisfatório para veranicos de 15 dias.

Utilizando os diagramas de dispersão para as relações observadas e RSM97, o coeficiente de determinação explicou melhor as regiões da Serra da Ibiapaba e do Setor Norte com dados do modelo regional.

No geral, o modelo regional subestima o número de veranicos em relação aos dados observados, tanto para o período de diagnóstico "previsão perfeita" quanto para o modo prognóstico forçado com a TSM persistida, o que indica que suas características físicas têm influência para quantificar a precipitação e sua distribuição espacial no Estado, além de conter uma topografia mais refinada, que favoreceu um melhor desempenho na variabilidade da precipitação.

Recomenda-se, para estudos futuros, uma análise com downscaling estatístico, ou com novos modelos dinâmicos regionais, como, também, com um maior número de anos de previsão para que se possam obter conclusões mais categóricas.

\section{Agradecimentos}

Os autores agradecem à Fundação Cearense de Meteorologia e Recursos HídricosFUNCEME pelos dados concedidos. Esses resultados fazem parte da Dissertação de Cláudia Patrícia Rickes no Mestrado Acadêmico de Ciências Físicas e Aplicadas da Universidade Estadual do Ceará-UECE.

\section{Referências}

ALVES, J. M. B. 2012. Recentes variações climáticas no Nordeste do Brasil com ênfase a precipitação: relações com ENOS, DIPOLO de TSM no Atlântico Tropical e a Oscilação Decadal do Pacífico. Revista de Geografia, v. 29, n. 3, p. 147-166.

ALVES, J. M. B.; FERREIRA, F. F.; CAMPOS, J. N. B. 2005. Movimento Vertical e Índices Atmosféricos Associados às Células Hadley e Walker em Anos de Contrastes Climáticos: Relação com as Chuvas no Setor Norte do Nordeste do Brasil. Revista Brasileira de Meteorologia, v. 20, n. 18, p. 15-36.

ALVES, J. M. B.; BRISTOT, G.; COSTA, A. A. A.; MONCUNNIL, D. F.; SILVA, E. M.; SANTOS, A. C. S.; BARBOSA, W. L; NÓBREGA, D. S. B.; FILHO, V. P. S.; SOUZA, ALVES, I. 2003. Uma aplicação da técnica de "downscaling" dinâmico no setor norte da região nordeste do Brasil. Revista Brasileira de Meteorologia, v. 18, n. 2, p. 161-180.

ALVES, J. M. B.; SOUZA, E. B.; REPELLI, C. A.; FERREIRA, N. S. 1997. Um estudo da variabilidade pluviométrica no setor leste do Nordeste brasileiro e a influência do fenômeno El Nino/Oscilação Sul. Revista Brasileira de Meteorologia, v. 12, n. 2, p. 25-39.

ALVES, J. M. B.; REPELLI, C. A. 1992. Variabilidade Pluviométrica no setor norte do Nordeste e os eventos EI Niño - Oscilação Sul. Revista Brasileira de Meteorologia, v. 7, n. 2, p. 583-592.

ANDREOLI, R. V.; KAYANO, M. T. 2007. A importância relativa do Atlântico Tropical Sul e Pacífico Leste na variabilidade de precipitação do Nordeste do Brasil. Revista Brasileira de Meteorologia, v. 22, n. 1, p. 63-74. 
HASTENRATH, S. 1984. Interannual variability and annual cycle: mechanisms of circulation and climate in the tropical Atlantic. Monthly Weather Review, v. 112, p. 1097-1107.

KOUSKY, V. E.; ROPELEWSKI, C. F. 1989. Extremes in the Southern Oscillation and their relationship to precipitation anomalies with emphasis on the South American region. Revista Brasileira de Meteorologia, v. 4, n. 1, p. 351-363.

LUCENA, D. B; SERVAIN, J.; GOMES FILHO, M. FR. 2011. Avaliação do Impacto de Eventos Climáticos Extremos nos Oceanos Pacífico e Atlântico sobre a Estação Chuvosa no Nordeste do Brasil. Revista Brasileira de Meteorologia, v. 26, p. 287-294.

LUCENA, D. B.; SERVAIN, J.; GOMES FILHO, M. F. 2010. Evolução do impacto de eventos climáticos extremos nos oceanos Pacifico e Atlântico sobre a estação Chuvosa no Nordeste do Brasil. In: JACQUES, S. (ED.); CAMPOS, J. N. B. (ED.); MARTINS, E. S. P. R. (ED.); REIS, D. S. (Ed.). (Org.). Clima do Atlântico Tropical e impactos sobre o Nordeste $($ CATIN $)=$ Climat de l'Atlantique Tropical et impactssurle Nordeste (CATIN). Fortaleza: FUNCEME/IRD, v. 1, p. 176-197.

MOURA, A. D.; SHUKLA, J. 1981. On the Dynamics of Droughts in Northeast Brazil: Observations, Theory, and Numerical Experiments with a General Circulation Model, J. Atmos. Sci., v. 38, n. 12, p. 2653-2675.

NOBRE, P.; MOURA, A. D.; SUN, L. 2001. Dynamical downscaling of seasonal climate prediction over Nordeste Brazil with ECHAM3 and NCEP Regional Spectral Model at IRI. Bull. Amer. Meteor. Soc., v. 82, p. 2787-2796.

NOBRE, P.; SHUKLA, J. 1996. Variations of Sea Surface Temperature, Wind stress, and rainfall over the tropical over the tropical Atlantic and South America. J. Climate, v. 9, n. 10, p. 24642479.

OLIVEIRA, G. S. 1999. O El Niño e você: o fenômeno climático. São José dos Campos, SP: TRANSTEC.

PEREIRA, J. M. R.; VASCONCELOS JÚNIOR, F. C.; MARTINS, E. S. P. R.; REIS JÚNIOR, D.
S.; NORONHA, A. W. T. 2015. Climate seasonal forecast and derived indexes for droughts in Ceará, Brazil. In: Joaquin Andreu Alvarez. (Org.). Drought: Research and Science-Policy Interfacing. 1ed. Londres: CRC Press, pp. 315320.

REPELli, C. A.; ALVES, J. M. B. 1996. A variabilidade interanual da estação chuvosa no Estado do Ceará e a probabilidade de ocorrência de veranicos. Revista Brasileira de Agrometeorologia, v. 4, n. 1, p. 107-118.

SERVAIN, J. 1991. Simple Climatic for the Tropical Atlantic Ocean and Some Applications. J. Geophys. Res, v. 96, n. C8, p. 15137-15146.

SOUZA, E. B; ALVES, J. M. B.; XAVIER, T. M. B. S. 1998. A estação chuvosa no semiárido nordestino nos anos de predominância de aquecimento ou resfriamento em toda bacia do Atlântico Tropical. Anais do congresso brasileiro de meteorologia, Ed. X. Brasília. 5p.

SUN, L.; MONCUNILL, D. F.; LI, H.; MOURA, A. D.; FILHO, F. A. S.; ZEBIAK, S. E. 2006. An operational dynamical downscaling prediction system for Nordeste Brazil and the 2002-04 realtime forecast validation. Journal of Climate, v. 19, p. 1990-2007.

SUN, L.; MOURA, A. D.; MONCUNILL, D. F.; Li, H.; FILHO, F. A. S. 2005. Climate Downscaling over Nordeste Brazil using NCEP RSM97. Journal of Climate, v. 18, p. 551-567.

SOUZA, E. B.; ALVES, J. M. B.; REPELLI, C. A. 1998. Influência dos Oceanos Pacífico e Atlântico na estação chuvosa do semiárido Nordestino. Revista Brasileira de Agrometeorologia, v. 6, n. 2, p. 277-283.

UVO, C. B. 1989. A Zona de Convergência Intertropical (ZCIT) e sua relação com a precipitação da Região Norte do Nordeste Brasileiro. (INPE - 4887 - TDL/378). Dissertação (Mestrado em Meteorologia), Instituto Nacional de Pesquisas Espaciais, São José dos Campos.

WITTE, R. S; WITTE, J. S. 2005. Estatística, $7^{\text {a }}$ edição, LTC, ISBN: 8521614411, Rio de JaneiroRJ. 